\title{
Local geometry of isoscalar surfaces
}

\author{
César Dopazo and Jesús Martín \\ Área de Mecánica de Fluidos, Universidad de Zaragoza, María de Luna 3, Zaragoza 50018, Spain \\ Juan Hierro \\ Laboratorio de Investigación en Tecnologías de la Combustión, CSIC, María de Luna 10, Zaragoza 50018, Spain
}

(Received 20 July 2007; published 27 November 2007)

\begin{abstract}
An inert dynamically passive scalar in a constant density fluid forced by a statistically homogeneous field of turbulence has been investigated using the results of a $256^{3}$ grid direct numerical simulation. Mixing characteristics are characterized in terms of either principal curvatures or mean and Gauss curvatures. The most probable small-scale scalar geometries are flat and tilelike isosurfaces. Preliminary correlations between flow and scalar small-scale structures associate highly curved saddle points with large-strain regions and elliptic points with vorticity-dominated zones. The concavity of the scalar profiles along the isosurface normal coordinate $x_{n}$ correlates well with negative mean curvatures, Gauss curvatures displaying any sign, which correspond to scalar minima, tiles, or saddle points; on the other hand, convexity along $x_{n}$ is associated with positive mean curvatures, Gauss curvatures ranging from negative to positive signs, featuring maxima, tiles, or saddle points; inflection points along $x_{n}$ correlate well with small values of the mean curvature and zero or negative values of $k_{g}$, corresponding to plane isosurfaces or saddle points with curvatures of equal and opposite signs. Small values of the scalar gradient are associated with elliptic points, either concave or convex $\left(k_{g}>0\right)$, for both concave and convex scalar profiles along $x_{n}$. Large values of the scalar gradient (or, equivalently, scalar fluctuation dissipation rates) are generally connected with small values of the Gauss curvature (either flat or moderate-curvature tilelike local geometries), with both concave and convex scalar profiles along $x_{n}$ equally probable. Vortical local flow structures correlate well with small and moderate values of the scalar gradient, while strain-dominated regions are associated with large values.
\end{abstract}

DOI: 10.1103/PhysRevE.76.056316

PACS number(s): 47.51.+a, 47.27.Gs, 47.27.tb

\section{INTRODUCTION}

Mixing of scalar fields, such as temperature and chemical species concentrations, is ubiquitous in energy generation and chemical processes, among other industrial and natural systems. Significant scalar fluctuations, induced by a chaotic velocity and/or injection devices, pose a difficult problem, commonly with very large spatial and temporal heterogeneities. The mathematical description of turbulent mixing often leads to stochastic simulations [1,2], with real physics playing a secondary role. Few attempts are registered of models mimicking mixing by appealing to intuitive and rather simplified physical descriptions [3].

While the structure of small scales of a turbulence field has been categorized in terms of its velocity gradient invariants [4], a similar methodology is missing for scalar fields. Gibson $[5,6]$ emphasized the importance in mixing of zero scalar-gradient points; he elucidates two-dimensional (2D) mechanisms for zero-gradient point generation by a turbulent field: (i) isolation from a region of uniform scalar gradient due to direct distortion by a convective velocity (turbulent eddy) stronger than the restoring diffusive velocity (this effect looks like a horseshoe map with stretching and folding [7]) and (ii) secondary splitting by local strain; their generation rate depends on the strain rates, on the initial size of the small-scale scalar structures, and on the diffusion coefficient.

Moffatt $[8,9]$ classifies the scalar field critical points (zero gradient) in terms of the eigenvalues of the scalar Hessian (second-derivative) tensor; depending on the index of the critical points, elliptic extremal points (maxima and minima) or saddle points of two types are encountered. Four possible ways to establish homoclinic separatrix connections for isoscalar surfaces through a saddle point are described. Topological transitions complicating a relatively simple, initially spherical, local scalar field with a single extremal point inside it are clearly illustrated. It is also stated that turbulence alone can generate neither extremal nor saddle points, requiring the cooperation of molecular diffusion. Spectral implications are explored.

Wang and Peters [10] investigate small-scale scalar statistics through the use of direct numerical simulation (DNS). The notion of "dissipation elements," convoluted regions over which two given extremal points are reached via scalar gradient trajectories, is introduced. Numerical results allow the extraction of typical element diameters of a few times the Kolmogorov microscale and lengths of the order of the Taylor microscale. A dissipation element is characterized in terms of the distance between two extremal points and their scalar value difference; a stochastic evolution equation for the probability density function of that distance is derived and numerically solved.

Dopazo et al. [11,12] use results from DNS to describe the local geometry of a reactive dynamically passive scalar in terms of the isosurface principal curvatures. Transport equations for the scalar gradient modulus and for the mean curvature permit analysis of the different contributions of turbulence, molecular diffusion, and chemical reaction. It is shown that molecular diffusion, both normal to isoscalar surfaces and tangential to them (curvature-induced diffusion), scalar dissipation, and chemical conversion are more intense in plane and tilelike (very small curvature in one spatial di- 
rection, and small, moderate, or large curvature in the orthogonal one) structures.

This paper is an attempt to improve upon the description of the local geometry of inert scalar fields in turbulence using DNS results. The mathematical tools are presented in Sec. II; a scalar field is locally characterized by its value, by its first and second derivatives in the normal direction to its corresponding isosurface, and by the curvature of the latter. The isocalar surfaces are classified in terms of the two nonzero-curvature tensor eigenvalues (principal curvatures) or, alternatively, in terms of its two nonzero invariants (mean, $k_{m}$, and Gauss, $k_{g}$, curvatures). The parabola $k_{g}=k_{m}^{2}$ separates the real from the excluded complex curvature regions. DNS corroborates both that the third curvature tensor invariant vanishes and that no points appear in the excluded region of a $\left(k_{m}, k_{g}\right)$-plane representation. Section III briefly describes the numerical method followed to obtain the DNS turbulence and scalar field results, which are reported and discussed in Sec. IV. Some concluding remarks and suggestions for future work are finally presented in Sec. V.

\section{MATHEMATICAL DESCRIPTION}

An isoscalar surface $Y(\mathbf{x}, t)=$ const evolves with time according to

$$
\frac{\partial Y}{\partial t}+\mathbf{u}^{Y} \cdot \nabla Y=0
$$

where $Y(\mathbf{x}, t)$ is the mass fraction of the scalar field, at point $\mathbf{x}$ and time $t, \mathbf{u}^{Y}$ is the absolute velocity of the isosurface and $\boldsymbol{\nabla}$ stands for the gradient operator. The isosurface normal velocity relative to the fluid, $V$, defined by

$$
V \mathbf{n}=\mathbf{u}^{Y}-\mathbf{u},
$$

is termed the propagation speed, $\mathbf{u}$ being the local fluid velocity and $\mathbf{n}$ the unit vector normal to the isosurface given by

$$
\mathbf{n}=\frac{\nabla Y}{|\nabla Y|}
$$

$|\nabla Y|$ denotes the modulus of $\boldsymbol{\nabla} Y$.

Equation (1) can, alternatively, be written as

$$
\frac{\partial Y}{\partial t}+\mathbf{u} \cdot \nabla Y=-V|\nabla Y| .
$$

Should the scalar $Y(\mathbf{x}, t)$ be convected and diffused, it obeys the transport equation

$$
\frac{\partial Y}{\partial t}+\mathbf{u} \cdot \nabla Y=\frac{1}{\rho} \boldsymbol{\nabla} \cdot(\rho D \nabla Y)
$$

where $\rho$ is the fluid density and $D$ stands for the molecular scalar diffusion coefficient. Equating the right sides of Eqs. (4) and (5), while, simultaneously, operating on the latter, one can readily obtain

$$
V=-\frac{1}{\rho} \mathbf{n} \cdot \boldsymbol{\nabla}(\rho D)-\frac{D}{|\nabla Y|} \mathbf{n} \cdot \nabla(|\nabla Y|)-D(\boldsymbol{\nabla} \cdot \mathbf{n}) .
$$

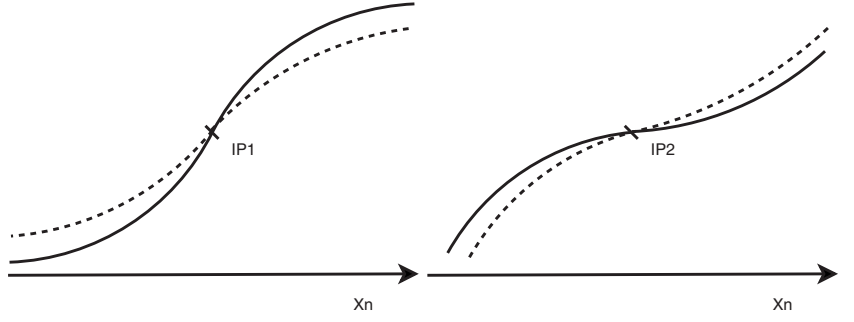

(a)

(b)

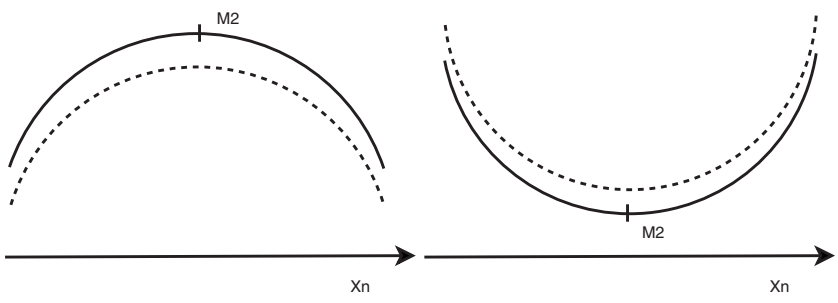

(c)

(d)

FIG. 1. Scalar profile in the normal direction to isosurfaces $Y\left(x_{n}, \ldots, t\right)$. Solid line at time $t$ and dashed line at time $t+\Delta t$. IP1, inflection point (concave-convex), IP2, inflection point (convexconcave), $M 1$ maximum point, and $M 2$ minimum point.

Let $x_{n}$ represent the local coordinate normal to the isosurface, growing in the positive direction of $\mathbf{n}$. Equation (6) can then be recast as

$$
V=-\frac{1}{\rho} \frac{\partial \rho D}{\partial x_{n}}-\frac{D}{\partial Y / \partial x_{n}} \frac{\partial^{2} Y}{\partial x_{n}^{2}}-D(\boldsymbol{\nabla} \cdot \mathbf{n}) .
$$

The first term on the right-hand side is the contribution of $\rho D$ variations normal to the isosurface to its propagation speed. For example, in a premixed system, with $Y$ equal to the reaction progress variable, the density decreases and the diffusion coefficient increases with $x_{n}$; therefore, this contribution to $V$ might, plausibly, be small compared to the other two terms. This contribution is zero for constant density, constant diffusion coefficient processes.

The second term in Eq. (7) is the normal propagation velocity of a flat front. The local profile of $Y(\mathbf{x}, t)$ in the direction of $x_{n}$ determines the propagation characteristics. Inflection points of the scalar field with nonzero gradients propagate only due to isosurface curvature. The conservation equation governing $Y(\mathbf{x}, t)$ becomes the unsteady heat conduction equation at extremal points. These extremal points move at an infinite speed relative to the fluid, which has no physical implications, as the product $-V|\boldsymbol{\nabla} V|$ is finite and equal to $\partial^{2} Y / \partial x_{n}^{2}$; extremal points become singularities, with neither a well-defined normal vector nor a propagation direction, which disappear instantaneously due to diffusion.

Mixing is visualized as a smoothing process of scalar fluctuations; deviations from the scalar mean are suppressed by molecular diffusion. The dissipation of scalar fluctuations takes place in the smallest scales. The one-dimensional $Y\left(x_{n}, t\right)$ local profile in the normal direction, shown in Fig. 1, about an inflection point can either smooth or sharpen de- 
pending on its local variation from concave to convex, or the other way round, along $x_{n}$; smoothing is equivalent to disappearance of small scales, or, equivalently, large wave numbers, while sharpening implies the generation of small structures as a previous step to smoothing. Sharpening inflection points shall evolve toward smoothing ones with time. Maxima with scalar content above the mean mass fraction and minima with values below it tend to move closer to the mean due to molecular diffusion; on the other hand, minima above the mean and maxima below it move away from the scalar mean. The complete picture of turbulent mixing is obtained by allowing the small-scale flow topologies interact with the previous scalar local geometries.

The third term in Eq. (7) is the curvature-induced speed. This contribution vanishes for flat fronts and saddle points with positive and negative principal curvatures of equal absolute value. Isosurfaces concave toward $\mathbf{n}$ imply $\boldsymbol{\nabla} \cdot \mathbf{n}<0$ and, therefore, a positive $V$; on the contrary, iso-scalar surfaces convex toward $\mathbf{n}$ mean $\boldsymbol{\nabla} \cdot \mathbf{n}>0$ and, thus, a negative $V$. Therefore, the curvature-induced propagation speed, due to molecular diffusion, of an isoscalar surface is always in the sense of its geometric concavity.

The conventional way to characterize the scalar fluctuation reduction process is by defining its dissipation rate. The transport equation for the square scalar fluctuation $Y^{\prime 2}$ $=(Y-\bar{Y})^{2}, \bar{Y}$ being the mean mass fraction, is

$$
\frac{\partial Y^{\prime 2}}{\partial t}+\mathbf{u} \cdot \nabla Y^{\prime 2}=D \nabla^{2} Y^{\prime 2}-2 D\left(\nabla Y^{\prime}\right)^{2}
$$

The right-hand side of Eq. (8) contains the diffusive transport and the dissipation of $Y^{\prime 2}$, respectively. The first contribution can be rewritten as

$$
D \boldsymbol{\nabla}^{2} Y^{\prime 2}=D \frac{\partial^{2} Y^{\prime 2}}{\partial x_{n}^{2}}+D(\boldsymbol{\nabla} \cdot \mathbf{n})\left|\frac{\partial Y^{\prime}}{\partial x_{n}}\right|,
$$

where the first term on the right-hand side is the diffusive transport normal to the isosurface, while the second one is the curvature-induced molecular diffusion, tangential to it. $\epsilon_{Y}=D\left(\nabla Y^{\prime}\right)^{2}$ is termed the scalar fluctuation dissipation rate; an equivalent expression for it is $\epsilon_{Y}=D\left(\partial Y^{\prime} / \partial x_{n}\right)^{2}$. Therefore, there is no contribution from curvature to the fluctuation dissipation rate.

The local geometry of the scalar field is thus defined by its value $Y(\mathbf{x}, t)$, by its first and second derivative, $\partial Y / \partial x_{n}$ and $\partial^{2} Y / \partial x_{n}^{2}$ in the direction normal to the isosurface, and also by its curvature. The latter is provided in detail by the curvature tensor, $\mathbf{n}_{i, j}=\partial n_{i} / \partial x_{j}$, which can be rephrased in terms of the scalar field derivatives as

$$
n_{i, j}=\frac{1}{|\boldsymbol{\nabla} Y|}\left(\delta_{i k}-n_{i} n_{k}\right) Y_{, k j} .
$$

$n_{i, j}$ is not a symmetric tensor. However, it admits row-side eigenvalues $k$ and eigenvectors $\mathbf{N}$, which can be obtained from the solution of the homogeneous system

$$
N_{i}\left(k \delta_{i j}-n_{i, j}\right)=0,
$$

which yields nonzero solutions if and only if

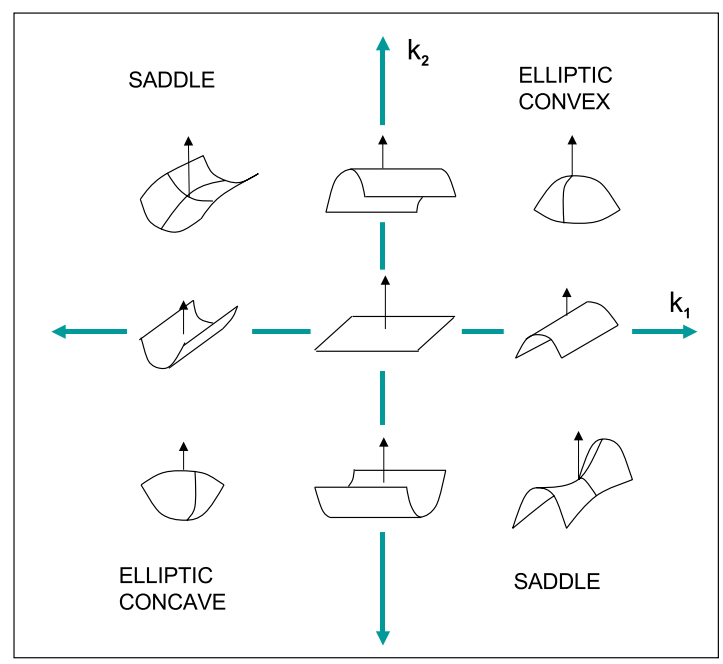

FIG. 2. (Color online) Classification of small-scale structures of isoscalar surfaces, in terms of their principal curvatures $k_{1}$ and $k_{2}$.

$$
\operatorname{det}\left\|k \delta_{i j}-n_{i, j}\right\|=0 .
$$

Equation (12) is equivalent to the cubic equation

$$
k^{3}+I_{1} k^{2}+I_{2} k+I_{3}=0 .
$$

The invariants of $n_{i, j}$ are defined as

$$
\begin{gathered}
I_{1}=-\boldsymbol{\nabla} \cdot \mathbf{n}, \\
I_{2}=(1 / 2)\left(n_{i, i} n_{j, j}-n_{i, j} n_{j, i}\right), \\
I_{3}=-\operatorname{det}\left\|n_{i, j}\right\| .
\end{gathered}
$$

$\mathbf{N}=\mathbf{n}$ is a solution to Eq. (12) as $n_{i} n_{i, j}=(1 / 2)\left(n_{i} n_{i}\right)_{, j}=0$, which, therefore, implies $k=0$ and, consequently, $I_{3}=0$. The relationship between $n_{i, j}$ and the intrinsic curvatures of isoscalar surfaces may be obtained from Weingarten's theorem [13].

With $I_{3}=0$, the cubic Eq. (13) reduces to a quadratic one,

$$
k^{2}+I_{1} k+I_{2}=0,
$$

whose solutions are

$$
k_{1}, k_{2}=\frac{-I_{1} \pm \sqrt{I_{1}^{2}-4 I_{2}}}{2} .
$$

$k_{1}$ and $k_{2}$ are the principal curvatures, $\left(k_{1}+k_{2}\right) / 2=-I_{1} / 2$ $=k_{m}$ is the mean curvature, and $k_{1} k_{2}=I_{2}=k_{g}$ stands for the Gauss curvature. The zone $k_{g}>k_{m}^{2}$ in the $k_{m}-k_{g}$ plane implies complex curvatures and, thus, it is excluded from study. DNS results corroborate both the presence of a zero third invariant of $n_{i, j}$ and the exclusion region of complex principal curvatures.

The different local geometries of isoscalar surfaces are depicted in Figs. 2 and 3 in terms of $k_{1}-k_{2}$ and $k_{m}-k_{g}$, respectively. 


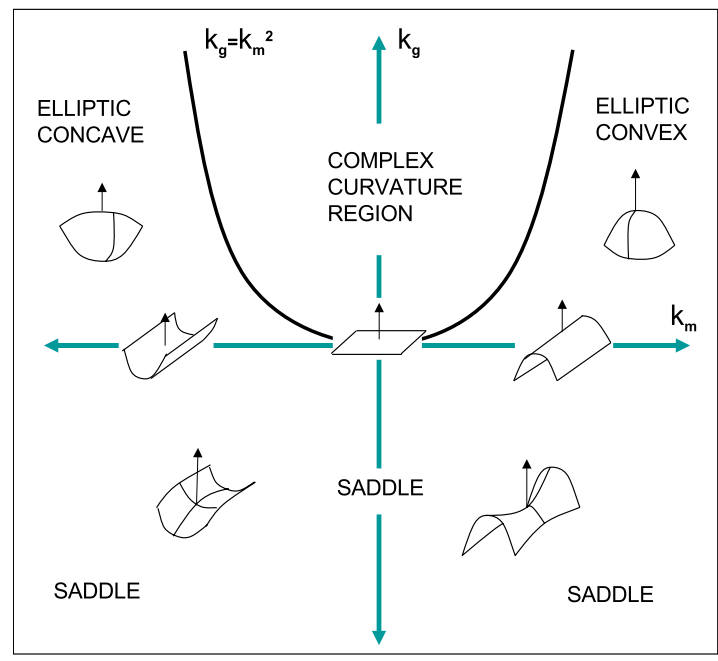

FIG. 3. (Color online) Classification of small-scale structures of isoscalar surfaces, in terms of their mean and Gauss curvatures $k_{m}$ and $k_{g}$.

\section{NUMERICAL METHOD}

A direct numerical simulation of a passive scalar field, whose fluctuations decay, in a forced, incompressible, isotropic, homogeneous, turbulent velocity field has been performed.

The evolution of the velocity field is governed by

$$
\begin{gathered}
\frac{\partial u_{i}}{\partial x_{i}}=0, \\
\frac{\partial u_{i}}{\partial t}+u_{j} \frac{\partial u_{i}}{\partial x_{j}}=-\frac{1}{\rho} P_{, i}+\nu u_{i, j j}+f_{i},
\end{gathered}
$$

where $f_{i}$ stands for the forcing term. Incompressibility implies that the pressure in Eq. (20) is not the thermodynamic pressure, assumed constant everywhere, but the hydrodynamic pressure (much smaller than the thermodynamic pressure but with nonzero gradient), whose only role is to project the evolution of the velocity field in a divergence-free manifold. The scalar field, in its turn, evolves according to Eq. (5) with $\rho D$ constant and a Schmidt number $\mathrm{Sc}=\nu / D=0.7$.

A pseudospectral numerical code [14-16] in the spatial domain with a second-order Runge-Kutta scheme in time has been chosen. The computational region is a cube of size $2 \pi$ with a $256^{3}$ grid and periodic boundary conditions. In order to reduce aliasing, a spherical filter is applied to data in Fourier space; all nodes corresponding to a wave number larger than $256 \times 0.471$ are set to zero. This filter is chosen [14] to suppress the double and triple aliasing contribution [17] caused by convective terms. Some amount of aliasing due to the single contribution of convective terms still remains; but it is neglected according to the criterion of Kida and Murakami [18], since the spatial resolution is such that the value of the largest wave number times the Kolmogorov scale is about 3.80. In order to resolve a field [19], it is sufficient for that product to be larger than unity; whereas it should be around 2 to resolve its gradient. The reason for choosing a value so high is imposed by the need of resolving curvature fields as well.

The time step has been chosen according to the Courant number criterion

$$
C=\frac{\Delta t}{\Delta x / \max \{\|\vec{u}\|\}}
$$

with $C=0.7$.

The forcing scheme has been that of Eswaran and Pope [20] with a zero correlation time of the forcing. To be more specific, all the Fourier-space nodes with a modulus less than $2 \sqrt{2}$, except the zero node which has no contribution, receive at each time step a random, white noise forcing contribution, where its phase is adjusted to enforce incompressibility and its intensity is such that the Reynolds number (based on the Taylor microscale) is close to 50 .

The integral length scale of the velocity field is less than one-sixth of the length of each edge of the computational box. This is well below the one-third value which guarantees the homogeneity of the resulting flow.

The velocity field is left to evolve first from an arbitrary initial spectrum until it reaches a statistically stationary situation, namely, an equilibrium between viscous dissipation and forcing.

An initial scalar field as close as possible to a homogeneously random distribution of 0 and 1 values is taken. This is realized by creating a random spectrum in Fourier space, transforming it into physical space, and setting a threshold such that values of the physical field below the threshold are set to 0 whereas those above the threshold are set to 1 . The threshold is selected so that the mean of this initial field is close to 0.5 and the wave numbers initially chosen for the random spectrum are those with modulus less than 4; which yields an initial scalar field with an initial integral length scale large enough to avoid rapid mixing. Then, the values of this field are smoothed, in Fourier space, to avoid strong numerical dispersion. In order to further reduce this problem, the full system is left to evolve for half a large-eddy turnover time before setting the initial condition of the simulation. In the end, the initial scalar field has a mean of 0.5 and a variance of 0.1701 (compare this with the variance of 0.25 that a fully unmixed field with the same mean should have).

Velocity fields are characterized by the Taylor-based Reynolds number $\left(\operatorname{Re}_{\lambda}\right)$, the integral length scale $\left(l_{\text {int }}\right)$, the length of the edge of the computational domain $(L=2 \pi)$, the viscosity ( $\nu=0.012$ in this simulation), the Taylor microscale $(\lambda)$, the Kolmogorov microscale $(\eta)$, the total kinetic energy $(\langle k\rangle)$, the kinetic energy dissipation rate $(\langle\epsilon\rangle)$, the root mean square velocity $\left(u_{\mathrm{rms}}\right)$ and the maximum wave number times the Kolmogorov microscale $\left(k_{\max } \eta\right)$.

Scalar fields are characterized by the diffusivity coefficient $(D=0.012 / 0.7=0.017143)$, the mean $(\langle Y\rangle)$, the variance $\left(\left\langle Y^{2}\right\rangle\right)$, and the scalar fluctuation dissipation rate $\left(\left\langle D Y_{, i} Y_{, i}\right\rangle\right)$.

Results have been analyzed at three different times: the initial condition when the scalar field has a mean close to 0.5 and a variance close to 0.17 ; when the scalar variance is 
TABLE I. Characteristic parameters of the studied DNS fields. Time is given in large-eddy turnover time units.

\begin{tabular}{lccc}
\hline \hline Time & 0 & 3 & 6 \\
\hline $\operatorname{Re}_{\lambda}$ & 46.5 & 47.6 & 53.02 \\
$\langle K\rangle$ & 2.61 & 2.49 & 2.91 \\
$\langle\epsilon\rangle$ & 1.75 & 1.52 & 1.67 \\
$u_{\text {rms }}$ & 1.32 & 1.29 & 1.39 \\
$l_{\text {int }}$ & 0.84 & 0.89 & 0.86 \\
$\lambda$ & 0.42 & 0.44 & 0.45 \\
$\eta$ & 0.032 & 0.033 & 0.032 \\
$k_{\max } \eta$ & 3.86 & 3.98 & 3.86 \\
$\langle Y\rangle$ & 0.500 & 0.500 & 0.500 \\
$\left\langle Y^{2}\right\rangle$ & 0.171 & 0.055 & 0.028 \\
$\left\langle D Y_{, i} Y, i\right.$ & 0.068 & 0.035 & 0.018 \\
\hline \hline
\end{tabular}

close to 0.055 , and, finally, when it is close to 0.028. Since the scalar field is chemically inert (passive), its mean value remains constant along the simulation.

In Table I, the characteristic parameters of the velocity and scalar fields for the three times are summarized.

\section{RESULTS}

Figure 4 displays the isoscalar surface $Y=0.5$, at time $t_{1}$. Zones with different values of the Gauss curvature $k_{g}$ have been drawn over the surface. The surface structure is complicated, and all the topological structures previously defined are present. Darker zones correspond to saddle points, while

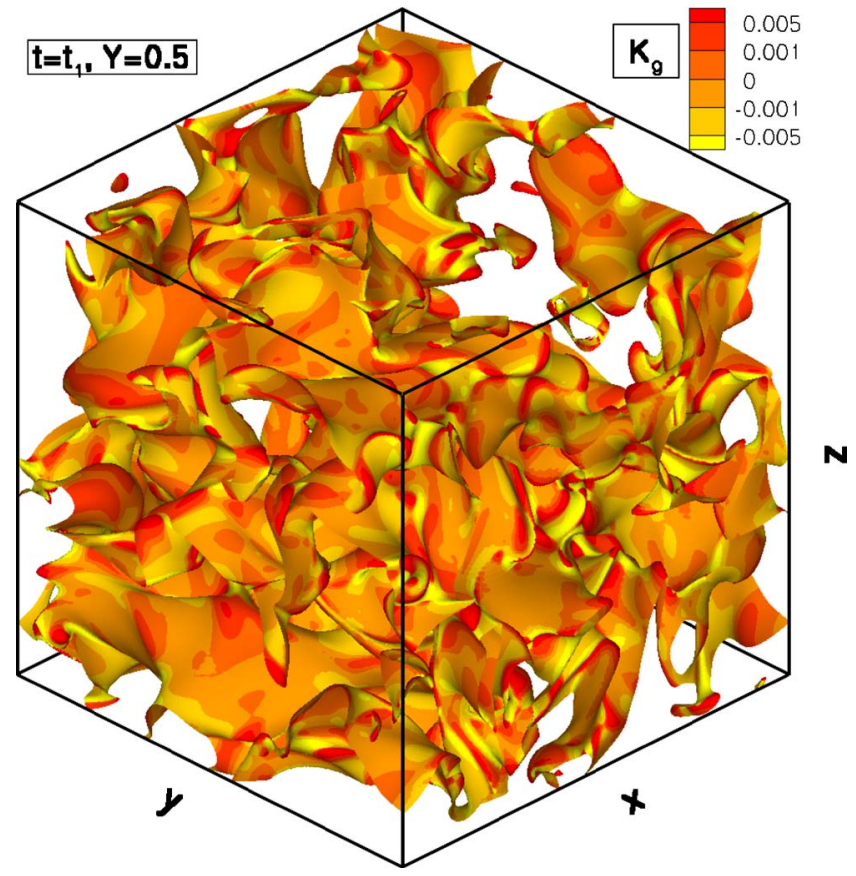

FIG. 4. (Color online) Simulation domain showing the isoscalar surface $Y=0.5$, at $t=t_{1}$. Zones with different values of the Gauss curvature $k_{g}$ have been drawn over the surface.

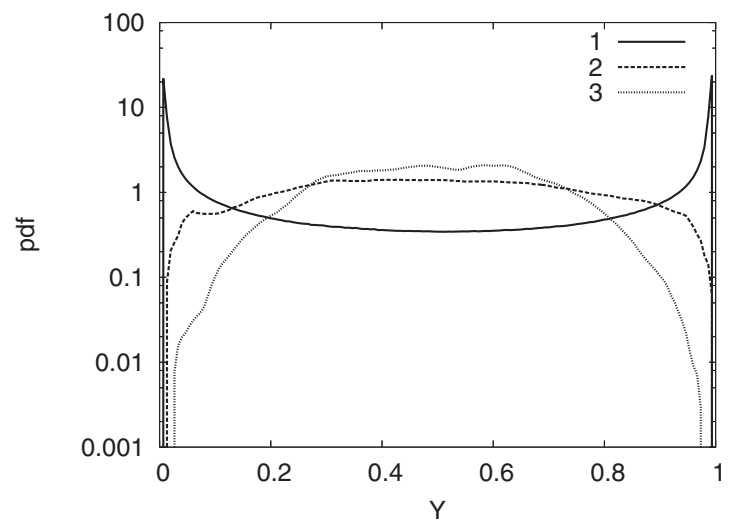

FIG. 5. PDF's of the mass fraction scalar $Y$ at the initial time (1), intermediate time (2), and final time (3) of the simulation.

the lighter ones indicate the convex or concave ones. Values close to zero denote flat or tilelike surfaces.

In Fig. 5, the probability density function (PDF) of the scalar mass fraction $Y$ at the initial, intermediate, and final simulation times are presented. Initially, the scalar distribution displays two peaks near the extreme values 0 and 1 , reminiscent of the starting partial segregation. For the intermediate time $t=t_{2}$ and the final time $t=t_{3}$, the mixing process progresses from a partially mixed situation to the initiation of a well-mixed system, tending asymptotically to a Gaussian distribution.

Figure 6 plots the PDF's of the curvature $\boldsymbol{\nabla} \cdot \mathbf{n}$, calculated for the scalar isosurfaces $Y=0.25,0.50$, and 0.75 at the intermediate time $t=t_{2}$. The PDF calculated for all the isosurfaces is also shown for comparison. A significant correlation between curvature and scalar value is apparent. For $Y=0.25$ the probability is displaced toward positive mean curvatures, corresponding to convex isosurfaces, while for $Y=0.75$ the skewness seems to be negative (concave surfaces). The PDF for $Y=0.50$ is approximately symmetric, similar to the one resulting for all scalar values, with convex and concave isosurfaces equally probable.

In Fig. 7 the total local isosurface propagation velocity $V$, as well as its normal $V_{n}$ and its curvature $V_{c}$ contributions, conditional upon the scalar value $Y$ at $t=t_{2}$, are presented.

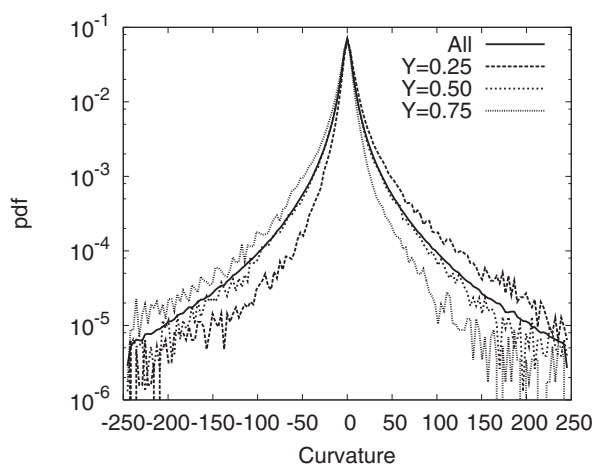

FIG. 6. PDF's of the curvature $\boldsymbol{\nabla} \cdot \mathbf{n}$ calculated for all the isosurfaces, compared to that calculated for $Y=0.25,0.50$, and 0.75 at the intermediate time $t=t_{2}$. 


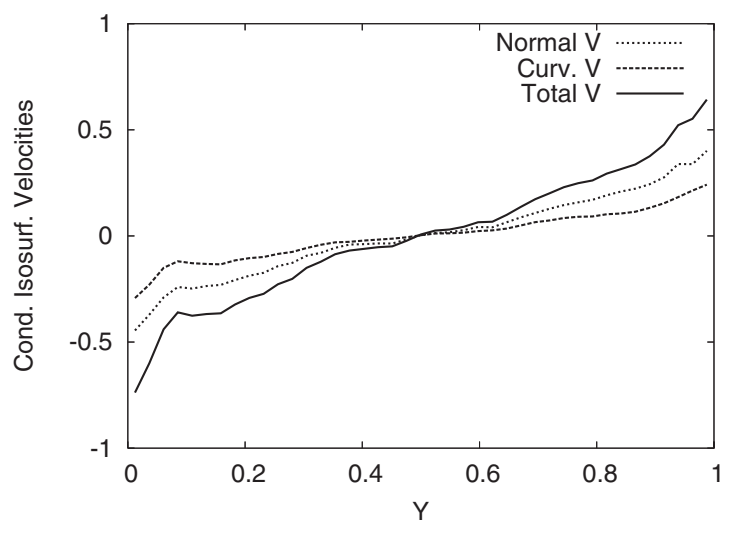

FIG. 7. Total local isosurface velocity $V$, its normal part $V_{n}$, and its curvature part $V_{c}$, conditional upon the scalar value $Y$ at time $t$ $=t_{2}$.

The total velocity $V$ appears clearly correlated with the scalar value $Y$; the dependence is approximately linear for small values of the scalar fluctuation $Y^{\prime}$, becoming nonlinear for large ones. The positive sign of that correlation agrees well with the negative one for the scalar diffusion, a consequence of the definition of $V$. Although the contribution of the normal part, $V_{n}$, is larger than that of the curvature, $V_{c}$, the latter is far from being negligible.

Figure 8 represents the joint PDF of the principal curvatures $k_{1}$ and $k_{2}$, calculated for all isosurfaces at $t=t_{2}$. Because of isotropy, the directions $x_{1}$ and $x_{2}$ are indistinguishable. $k_{1}$ is here taken as the larger of the two curvatures, and therefore all sample points will be below the line $k_{2}=k_{1}$, excluding the occurrence of pairs $\left(k_{1}, k_{2}\right)$ above it. The distribution seems symmetric with respect to the line $k_{2}=-k_{1}$, presents a maximum near the origin, and a high probability with long tails extending along the axes. The width of those tails decreases as the curvature magnitude increases. Consequently,

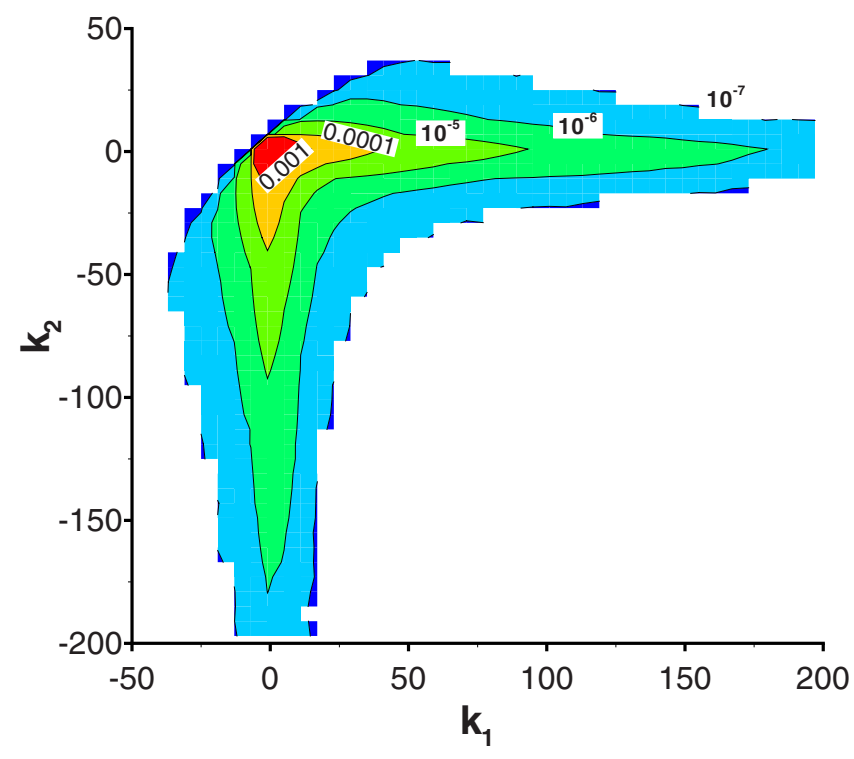

FIG. 8. (Color online) Joint PDF of the main curvatures $k_{1}$ and $k_{2}$ for all isosurfaces at the intermediate time $t=t_{2}$.

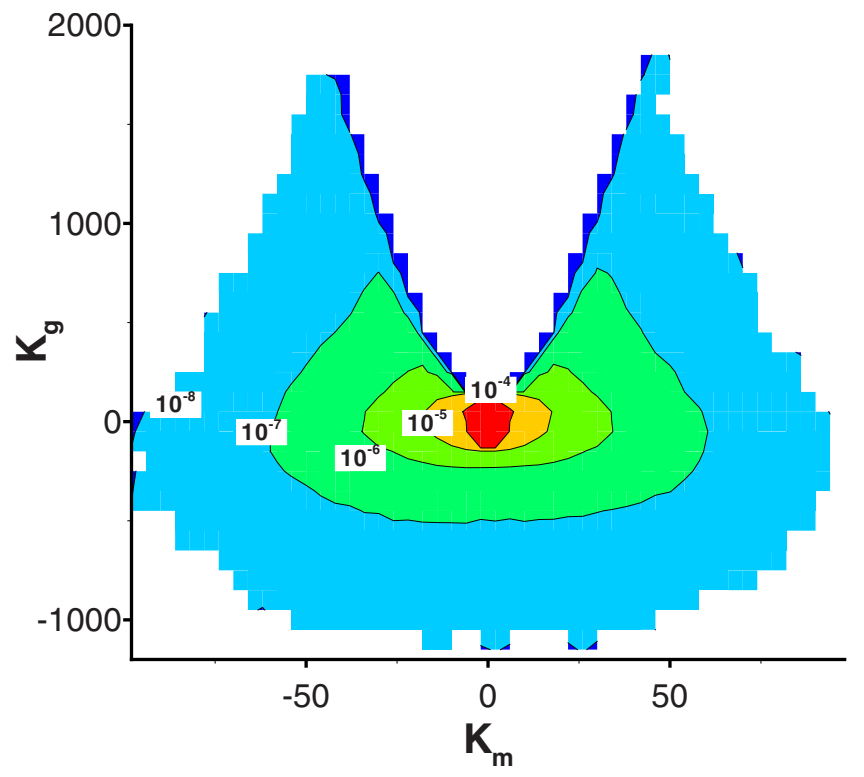

FIG. 9. (Color online) Joint PDF of the mean and Gauss curvatures $k_{m}$ and $k_{g}$, for all isosurfaces. $t=t_{2}$.

the most probable local structures are flat and saddle points with small curvatures. For moderate and large curvatures, saddles, and elliptic (concave and convex) or tile structures can occur, with a significantly larger probability of tiles.

Figure 9 depicts the joint PDF of the mean and Gauss curvatures $k_{m}$ and $k_{g}$, calculated for all isosurfaces at $t=t_{2}$. The resulting distribution is symmetric with respect to the $k_{g}$ axis, and displays a maximum below the origin, near to it. All samples are below the parabola $k_{g}=k_{m}^{2}$, separatrix of the prohibited region corresponding to complex curvatures. Isocontours surround the origin spreading over increasing areas as the curvature magnitude increases. Furthermore, the distribution seems skewed toward large positive values of $k_{g}$, and the contours present long tails on both sides of the limit curve $k_{g}=k_{m}^{2}$. This last feature is enhanced as largerpositive or negative-values of $k_{m}$ are reached. The description of local geometries in terms of $k_{m}-k_{g}$ is totally analogous to that provided by Fig. 8 .

Figure 10 is a plot of the scalar value $Y$ conditional upon the mean and Gauss curvatures for all isosurfaces at time $t$ $=t_{2}$. The correlation, previously observed in Fig. 6 between scalar and the curvature values, is clearer here; scalar values above the mean correspond to negative mean curvature, while negative scalar fluctuations are associated with positive $k_{m}$. In turn, for large positive $k_{m}$ values, $Y$ increases with decreasing $k_{g}$, and for large negative $k_{m}$ values the magnitude of $Y$ increases as $k_{g}$ does. Minimum scalar values occur for elliptic convex structures, intermediate values for saddle points, and maxima for elliptic concave ones.

The absolute value of the scalar gradient, $|\nabla Y|$, conditional upon the mean and Gauss curvatures, for all isosurfaces, at time $t=t_{2}$, is shown in Fig. 11. Equivalently, this figure provides the features of the scalar fluctuation dissipation rate. The resulting conditional values are symmetric with respect to the $k_{g}$ axis. The largest scalar gradients correlate with small values of both curvatures and for $k_{g}$ nega- 


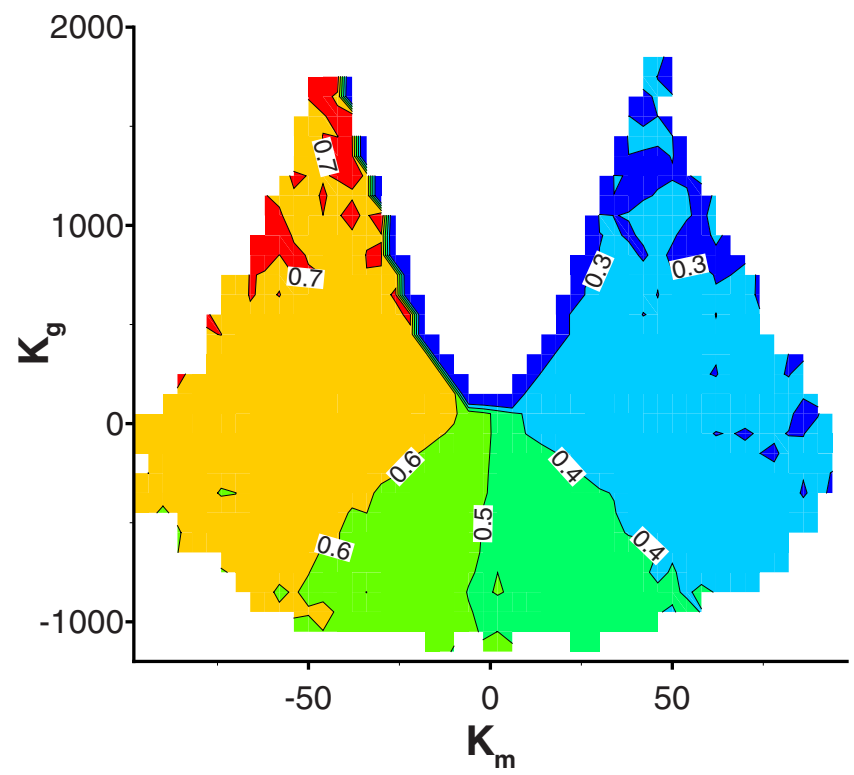

FIG. 10. (Color online) The scalar value $Y$ conditional upon the mean and Gauss curvatures $k_{m}$ and $k_{g}$, for all isosurfaces. $t=t_{2}$.

tive. Intermediate scalar gradients appear in a region surrounding the origin which extends along the $k_{m}$ axis, reaching moderate values, with only small relative values of $k_{g}$. As the scalar gradient magnitude decreases, large negative and positive values of $k_{g}$ are involved. Therefore, it seems that the scalar gradient magnitude has the following properties.

(i) It does not depend on the mean curvature sign.

(ii) It displays large values for zones of the isoscalar surfaces with small curvature. In previous experimental and DNS work [21-23], it was found that scalar dissipation rate maxima are organized in sheets; however, these maximum

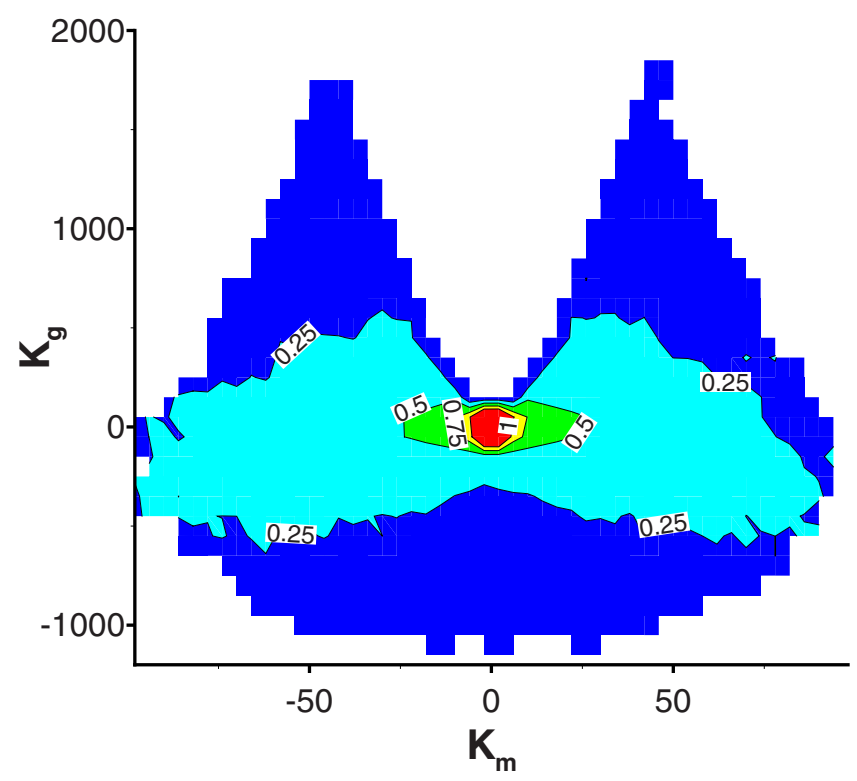

FIG. 11. (Color online) Modulus of the scalar gradient, $|\nabla Y|$, conditional upon the mean and Gauss curvatures, $k_{m}$ and $k_{g}$, for all isosurfaces at time $t=t_{2}$.

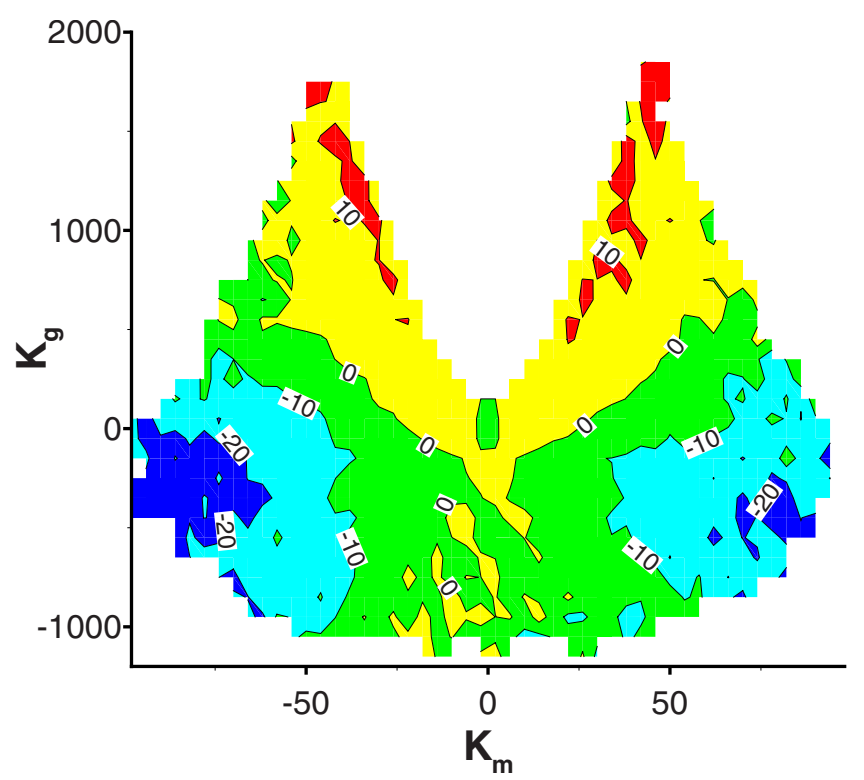

FIG. 12. (Color online) Second invariant of the velocity gradient tensor, $Q=\omega^{2} / 4-S_{i j} S_{i j} / 2$, conditional upon the mean and Gauss curvatures $k_{m}$ and $k_{g}$, at time $t=t_{2}$.

dissipation rate sheets are not necessarily coincident with isoscalar surfaces.

(iii) It has moderate and small values when $k_{m}$ and $k_{g}$ take large values, independently of their signs.

In order to explore the possible relationship between isoscalar surface structures and small-scale flow motions, the conditional average of $Q$, the second invariant of the velocity gradient tensor $\partial u_{i} / \partial x_{j} \equiv A_{i j}$, is plotted in Fig. 12 as a function of $k_{m}$ and $k_{g}$. For a constant density flow $Q=-A_{i j} A_{j i} / 2$ can be rephrased as $Q=\omega^{2} / 4-S_{i j} S_{i j} / 2$, where $S_{i j}$ is the strain rate tensor and $\omega$ is the vorticity. Hence, $Q$ is a measure of the local balance between vorticity and strain at every point in the flow. The figure shows a clear correlation of $Q$ with the curvature. For negative $k_{g}$ curvatures (saddle points) extreme values of $k_{m}$ appear related with intense strain $(Q$ $<0$ ), while points with large positive $k_{g}$ (elliptic concave or convex points) are related with focal motions, corresponding to dominant vorticity $(Q>0)$. On the contrary, little correlation is found for points having small or moderate curvatures-most of the samples-which display very low values of $Q$, without clear trends. Flat and tilelike scalar isosurfaces are typically immersed in varying strain regions from zero to large deformation rates.

Figure 13 depicts the joint PDF of the mean and Gauss curvatures corresponding to the isosurfaces $Y=0.25$ (top), 0.50 (center) and 0.75 (bottom) at time $t=t_{2}$. The result is consistent with the picture pointed out in Fig. 6 for the PDF of the curvature $\boldsymbol{\nabla} \cdot \mathbf{n}$. The plot for $Y=0.25$ indicates that scalar values below the mean better correlate with positive $k_{m}$ values, with enhanced amplitudes-positive or negative- of $k_{g}$. The opposite is true for values of $Y$ above its mean, as the distribution for $Y=0.75$ shows preference for negative mean curvatures, also with more probability of large $k_{g}$ values. The isosurface for the scalar value $Y=0.5$ (the mean) displays a symmetric joint PDF analogous to that 

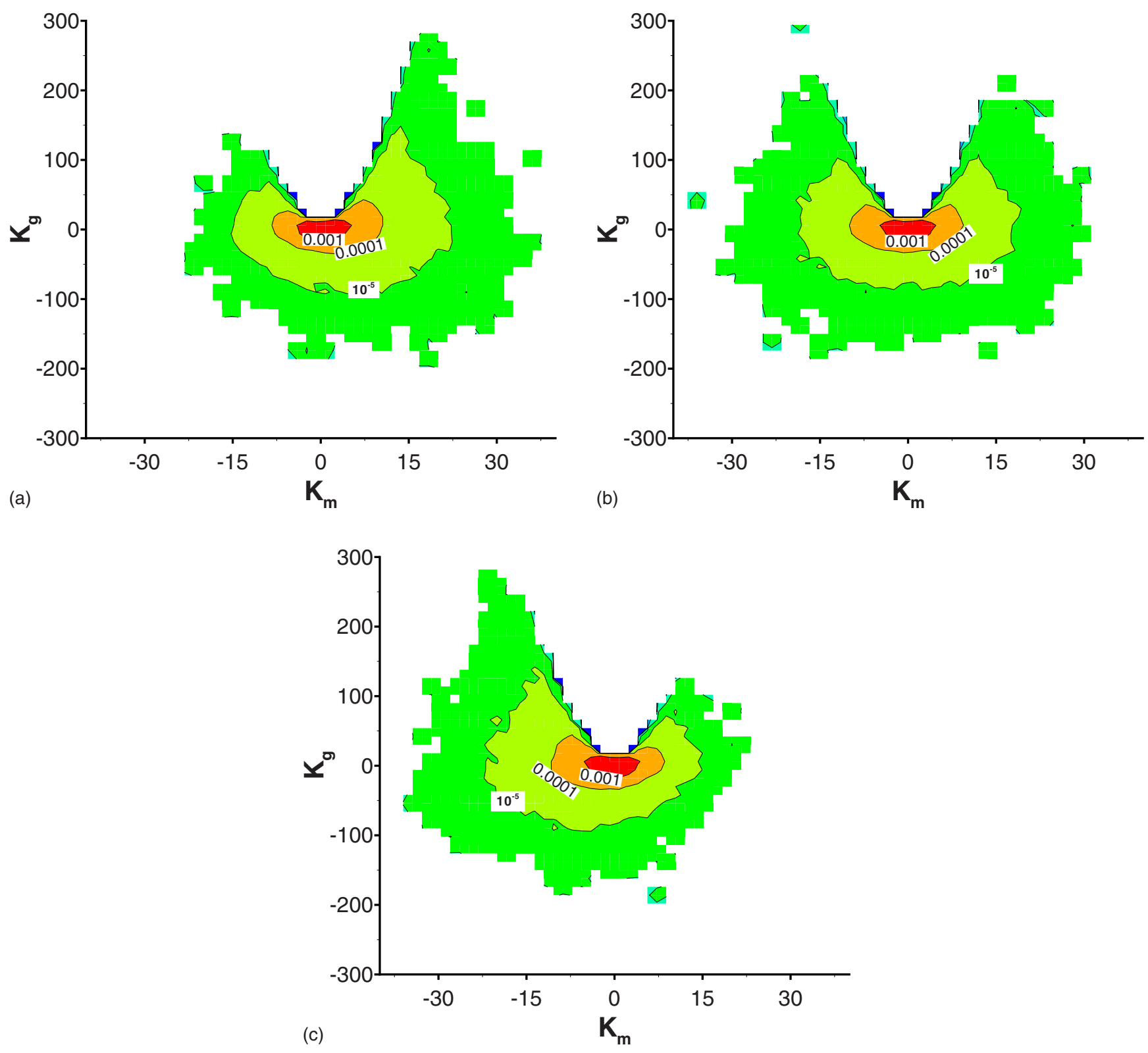

FIG. 13. (Color online) Joint PDF of the mean and Gauss curvatures $k_{m}$ and $k_{g}$, corresponding to the isosurfaces $Y=0.25$ (top), 0.50 (center), and 0.75 (bottom) at time $t=t_{2}$.

obtained for all scalar values in Fig. 9. The result confirms the previously noted trend of the isoscalar surfaces with negative fluctuation to present dominant positive curvature $\left(k_{1}+k_{2}>0\right)$ convex toward the scalar gradient direction, while positive scalar fluctuation isosurfaces present a predominantly concave shape $\left(k_{1}+k_{2}<0\right)$ toward the scalar gradient.

$D \partial^{2} Y / \partial x_{n}^{2}$ is the part of the scalar diffusion term that accounts for the molecular transport in the normal direction to the local isoscalar surface. Figure 14 depicts the normal second derivative as a function of the curvatures $k_{m}$ and $k_{g}$, calculated for the three isosurfaces $Y=0.25,0.50$, and 0.75 at time $t=t_{2}$. A clear correlation of $\partial^{2} Y / \partial x_{n}^{2}$ with the mean curvature $k_{m}$ is observed for $Y=0.5$ (the scalar mean); normal diffusion shares the sign of $k_{m}$, with the magnitude of the second derivative slightly increasing as large absolute values of $k_{m}$ are reached; this increase is higher for small values of $k_{g}$. This trend extends for the isosurfaces $Y=0.25$ and 0.75 , but predominantly positive values of $\partial^{2} Y / \partial x_{n}^{2}$ are found in the former, and negative in the latter. The zero isocontour appears slightly displaced toward negative values of $k_{m}$ for $Y=0.25$, and toward positive $k_{m}$ values for $Y=0.75$. This result seems logical, as normal diffusion behaves similarly to total diffusion, which has, generally, opposite sign to that of scalar fluctuation, as previously observed in Fig. 7.

Alternatively, $\partial^{2} Y / \partial x_{n}^{2}$ also represents the scalar annihilation, $\partial Y / \partial t$, of extremal points at a fixed spatial location. The material derivative of $Y$ at inflection points is given by $D(\boldsymbol{\nabla} \cdot \mathbf{n})|\boldsymbol{\nabla} Y|$. Figure 15(a) provides information on the joint PDF of $\partial^{2} Y / \partial x_{n}^{2}$ and $|\nabla Y|$; isoprobability contours are approximately ellipses elongated along the $|\nabla Y|$ axis. Maxima and minima are equally probable; inflection points with 

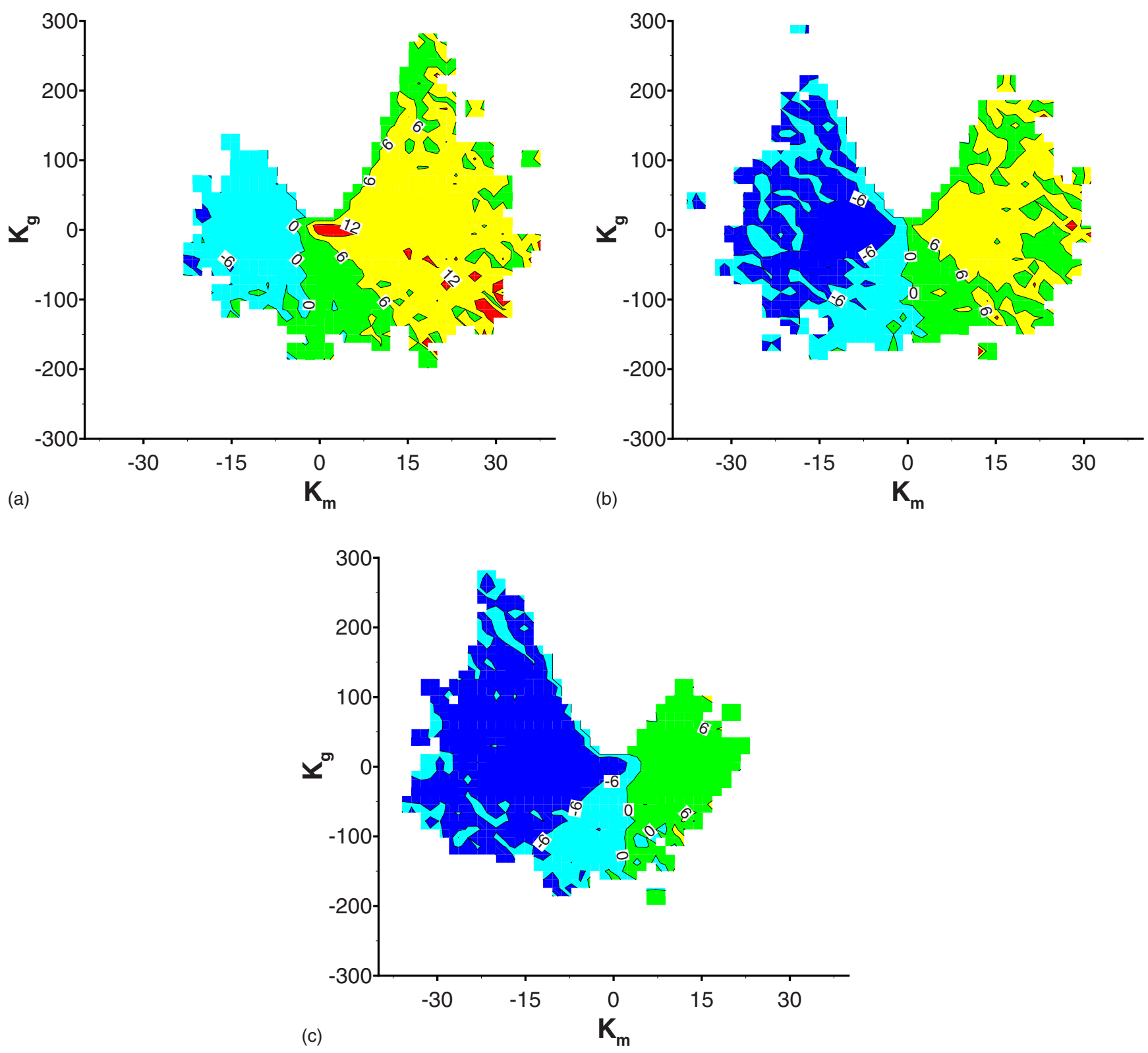

FIG. 14. (Color online) Second derivative of the scalar $Y$ in the normal direction, $\partial^{2} Y / \partial x_{n}^{2}$, conditional upon the mean and Gauss curvatures $k_{m}$ and $k_{g}$, corresponding to the isosurfaces $Y=0.25$ (top), 0.50 (center), and 0.75 (bottom) at time $t=t_{2}$.

small values of $|\nabla Y|$ or, equivalently, scalar fluctuation dissipation rate are more probable. Figure 15(b) indicates that convex isosurfaces, with positive mean curvature, are associated with positive second normal derivatives and decay with increasing $|\nabla Y|$. Figure 15(c) shows that elliptic points are correlated with small values of $\partial^{2} Y / \partial x_{n}^{2}$ and $|\nabla Y|$, while saddle points coincide with large positive and negative values of $\partial^{2} Y / \partial x_{n}^{2}$ with small and moderate $|\nabla Y|$. Large values of $|\nabla Y|$ generally imply flat and tilelike isosurfaces. Figure 15(d) indicates that vorticity-dominated regions coincide with both small values of $\partial^{2} Y / \partial x_{n}^{2}$ and small and moderate $|\nabla Y|$. Moderate and large values of $\partial^{2} Y / \partial x_{n}^{2}$ are associated with large $|\nabla Y|$ in strain-controlled zones.

\section{CONCLUSIONS AND FUTURE WORK}

Results of a $256^{3}$ grid DNS for an inert dynamically passive scalar in a constant density fluid forced by a statistically homogeneous field of turbulence have been used to describe mixing characteristics in terms of either principal curvatures or mean and Gauss curvatures. The predominant small-scale scalar geometries are apparently flat and tilelike isosurfaces. Large values of the scalar gradients are also associated with these structures. Preliminary correlations between flow and scalar small-scale structures are obtained, associating highly curved saddle points with large-strain regions and extremal points with vorticity-dominated zones. The curvatures for different isosurfaces moderately change from large to small scalar values. Concavity along the isosurface normal coordi- 

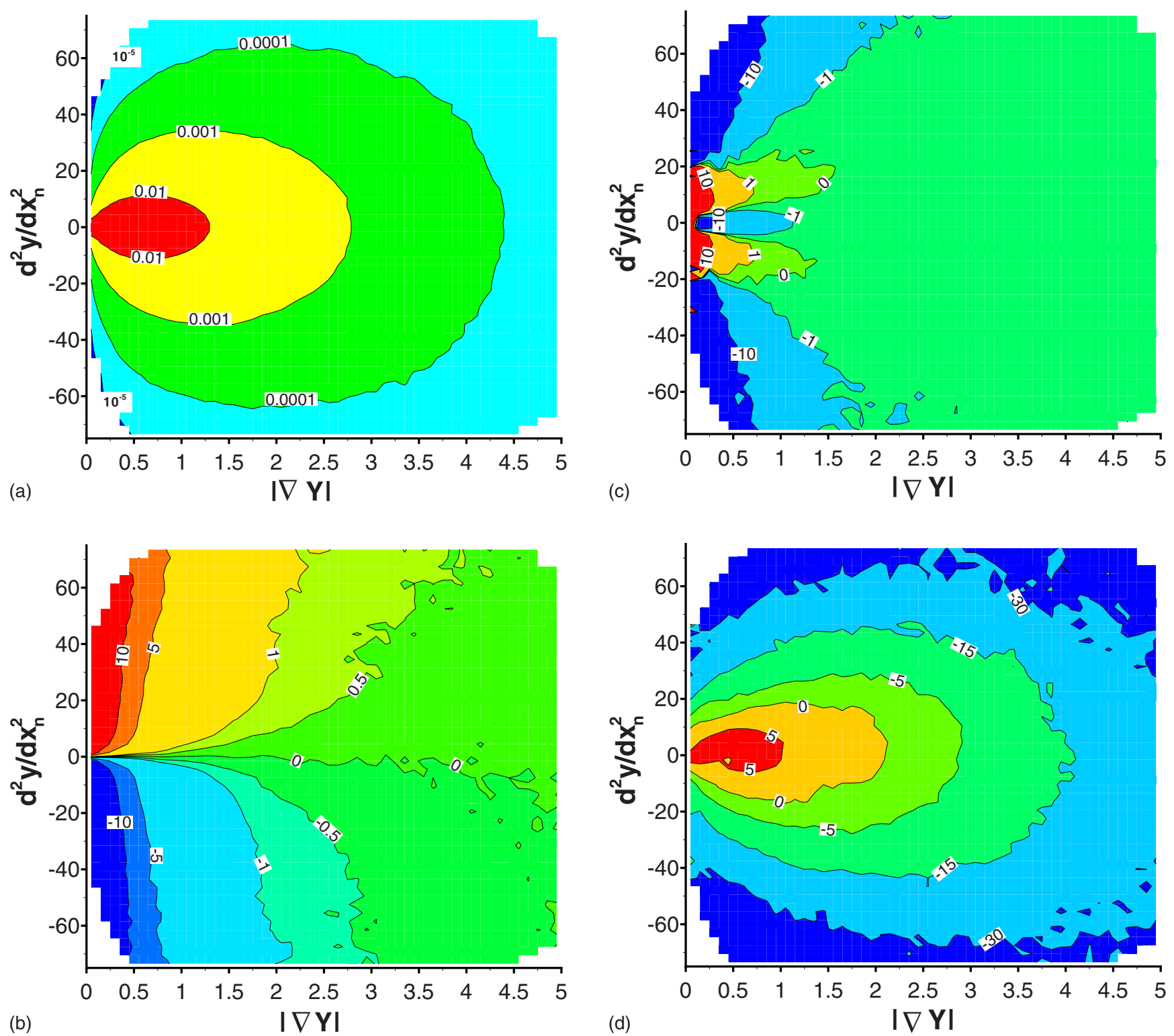

FIG. 15. (Color online) (a) Joint PDF of $\partial^{2} Y / \partial x_{n}^{2}$ and $|\nabla Y|$; (b) the mean curvature $k_{m}$; (c) the Gauss curvature $k_{g}$; and (d) the second invariant of the velocity gradient tensor $Q$, in terms of $\partial^{2} Y / \partial x_{n}^{2}$ and $|\nabla Y|$. Results are calculated for all scalar values at time $t=t_{2}$.

nate $\left(x_{n}\right)$ correlates well with negative mean curvatures, the Gauss curvature displaying any sign, corresponding to scalar minima, tiles, or saddle points; on the other hand, convexity along $x_{n}$ is associated with positive mean curvatures and Gauss curvatures ranging from negative to positive signs, connected to maxima, tiles, or saddle points; inflection points along $x_{n}$ correlate well with small values of the mean curvature and zero or negative values of $k_{g}$, corresponding to plane isosurfaces or saddle points with principal curvatures of equal and opposite signs. Small values of the scalar gradient are associated with elliptic points, either concave or convex $\left(k_{g}>0\right)$, and both concave and convex scalar profiles along $x_{n}$. As already mentioned, large values of scalar gradients are generally connected with small values of the Gauss curvature (either flat or tilelike with moderate-curvature local geometries) and both concave and convex scalar profiles along $x_{n}$ equally probable. Vortical local flow structures correlate well with small and moderate values of the scalar gradient, while strain-dominated regions are associated with large values.

Higher-resolution DNS's, increasing both the number of nodes and, possibly, the Reynolds number, would be necessary in order to improve the curvature statistics quality, since the study of the topology of isoscalar surfaces needs a better resolution than that required for studying gradient fields. The present research should also be extended to consider the time evolution of the isosurface geometry statistics at the different stages of mixing. The relationship between the small scales of the turbulent flow and those of the scalar field should be explored in depth. It would also be interesting to check the degree of alignment between maximum dissipation rate sheets and sheetlike (small-curvature) regions of isoscalar surfaces. Investigations of isoscalar surface local geometry in variable density turbulence, relevant to combustive flows, would be of great interest. Last, though not least, the impli- 
cations of scalar local structures for molecular mixing models should be scrutinized, mainly to try to include possible curvature effects.

Some stochastic mixing simulations involve the estimation of $D \nabla^{2} Y$ evaluated at specific isosurfaces $Y=\Gamma$; the decomposition of Eq. (7) of the diffusive term into two flat front plus one curvature contributions will, hopefully, help to unveil the relative importance of the three effects in turbulent combustion systems. Preliminary results for constant density turbulent reacting flows tend to indicate that high reaction rates occur at scalar structures with large curvatures only in one direction.

\section{ACKNOWLEDGMENTS}

The authors would like to thank Professor L. J. Boya of the University of Zaragoza for some interesting discussions on curvature tensor invariants. A part of this work has been performed under the auspices of the INTAS project 353 of the European Union. Juan Hierro would like to thank the Spanish Government and the Fulbright Commission for their support to spend one year at the Johns Hopkins University. The financial support of the DGA to the Computational Fluid Mechanics Group of the University of Zaragoza/LITEC is also acknowledged.
[1] S. Pope, Prog. Energy Combust. Sci. 11, 119 (1985).

[2] C. Dopazo, in Turbulent Reactive Flows, edited by P. Libby and F. Williams (Academic Press, New York, 1994), Chap. 7.

[3] A. Kerstein, J. Fluid Mech. 392, 277 (1999).

[4] J. Soria, M. Chong, R. Sondergaard, A. Perry, and B. Cantwell, Phys. Fluids 6, 871 (1994).

[5] C. Gibson, Phys. Fluids 11, 2305 (1968).

[6] C. Gibson, Phys. Fluids 11, 2316 (1968).

[7] J. M. Ottino, The Kinematics of Mixing: Stretching, Chaos, and Transport (Cambridge University Press, Cambridge, U.K., 1989).

[8] H. Moffatt, in New Trends in Turbulence, edited by M. Lesieur, A. Yaglom, and F. Davis (Springer, Berlin, 2001), pp. 319-340.

[9] H. Moffatt, in IUTAM Symposium on Geometry and Statistics of Turbulence, edited by R. Ricca (Kluwer, Dordrecht, 2001), pp. 13-22.

[10] L. Wang and N. Peters, J. Fluid Mech. 554, 457 (2006).

[11] C. Dopazo, J. Martín, J. Hierro, and J. Chen, in Simplicity, Rigor and Relevance in Fluid Mechanics. A Volume in Honor of Amable Liñán, edited by F. Higuera, J. Jiménez, and J. Vega (CIMNE, Barcelona, 2004).
[12] C. Dopazo, J. Martín, and J. Hierro, C. R. Mec. 334, 483 (2006).

[13] R. Aris, Vectors, Tensors and the Basic Equations of Fluid Mechanics (Prentice-Hall, Englewood Cliffs, NJ, 1962).

[14] S. Orszag and G. Patterson, in Lecture Notes in Physics Vol. 12, edited by M. Rosenblatt and Van Atta (Springer, Berlin, 1972), p. 127.

[15] R. Rogallo and P. Moin, Annu. Rev. Fluid Mech. 16, 99 (1984).

[16] V. Eswaran and S. Pope, Phys. Fluids 31, 506 (1988).

[17] R. Rogallo, Ames Research Center-NASA Technical Report No. TM-81315, 1981 (unpublished).

[18] S. Kida and Y. Murakami, Fluid Dyn. Res. 4, 347 (1989).

[19] J. Jiménez, A. Wray, P. Saffman, and R. Rogallo, J. Fluid Mech. 255, 65 (1993).

[20] V. Eswaran and S. Pope, Comput. Fluids 16, 257 (1988).

[21] L. K. Su and N. T. Clemens, J. Fluid Mech. 488, 1 (2003).

[22] J. Schumacher, K. R. Sreenivasan, and P. K. Yeung, J. Fluid Mech. 531, 113 (2005b).

[23] D. Kushnir, J. Schumacher, and A. Brandt, Phys. Rev. Lett. 97, 124502 (2006). 